\title{
Chiral excitonic instability of two-dimensional tilted Dirac cones
}

\author{
Daigo Ohki $\odot,{ }^{1, *}$ Michihiro Hirata $\odot,{ }^{2, \dagger}$ Takehiro Tani, ${ }^{1}$ Kazushi Kanoda $\odot,{ }^{3}$ and Akito Kobayashi ${ }^{1}$ \\ ${ }^{1}$ Department of Physics, Nagoya University, Chikusa-ku, Nagoya 462-8602, Japan \\ ${ }^{2}$ Institute for Materials Research, Tohoku University, Aoba-ku, Sendai 980-8577, Japan \\ ${ }^{3}$ Department of Applied Physics, University of Tokyo, Bunkyo-ku, Tokyo 113-8656, Japan
}

(Received 15 April 2020; revised 29 August 2020; accepted 2 September 2020; published 24 September 2020)

\begin{abstract}
The Coulomb interaction among massless chiral particles harbors unusual emergent phenomena in solids beyond the conventional realm of correlated electron physics. An example of such an effect is excitonic condensation of interacting massless Dirac fermions, which drives spontaneous mass acquisition and whose exact nature remains actively debated. Its precursor fluctuations growing prior to the condensate have been suggested by a recent nuclear magnetic resonance study in an organic material, hosting a pair of two-dimensional (2D) tilted Dirac cones at charge neutrality. Here, we theoretically study the excitonic transition in 2D tilted cones to understand the electron-hole pairing instability as functions of temperature $(T)$, chemical potential $(\mu)$, and in-plane magnetic field $(H)$. By solving a gap equation within a weak-coupling treatment and incorporating self-energy effects due to the Coulomb interaction through a renormalization-group technique, we calculate excitonic instability in a $T-\mu-H$ parameter space, and find that the pairing is promoted as $H$ is increased but suppressed as $\mu$ moves away from the charge-neutrality point. We show that these findings are explained by enhanced or degraded Fermi-surface nesting between the Zeeman-induced pockets connecting the two tilted cones. Furthermore, to evaluate the precursor excitonic fluctuations in relation to this diagram, we consider the Coulomb interaction via a ladder-type approximation and calculate the nuclear spin-lattice relaxation rate, which provides rational ways to understand otherwise puzzling experimental results in the organic material by the $\mu$ and $H$ dependence of the instability.
\end{abstract}

DOI: 10.1103/PhysRevResearch.2.033479

\section{INTRODUCTION}

The notable electronic properties of graphene as well as topological semimetals and insulators have been attracting increasing attention not only because of their exotic topological nature, but also due to their unusual effects induced by the electron-electron Coulomb interaction [1-7]. Unlike in conventional solids, the metallic screening in these systems is absent when the Fermi energy $E_{\mathrm{F}}$ is fixed at band-crossing points due to a vanishingly small density of states, resulting in preservation of the long-range part of the Coulomb interaction $[1,2]$. The strength of the interaction is then characterized by a dimensionless coupling constant $\alpha=e^{2} / 4 \pi \varepsilon_{0} \varepsilon \hbar v$ that is proportional to the ratio of the Coulomb potential to the electron kinetic energy, where $e$ is the elementary charge and $\varepsilon$ is the relative permittivity. This notable long-range form of the interaction causes an anomalous upward renormalization of the Fermi velocity $v$ by a self-energy effect, akin to what has been commonly discussed in the relativistic Dirac and

\footnotetext{
*Corresponding author: dohki@s.phys.nagoya-u.ac.jp

${ }^{\dagger}$ Corresponding author: michihiro_hirata@imr.tohoku.ac.jp
}

Published by the American Physical Society under the terms of the Creative Commons Attribution 4.0 International license. Further distribution of this work must maintain attribution to the author(s) and the published article's title, journal citation, and DOI.
Weyl theories [3,4]. Indeed, its influence in solids has been extensively analyzed for weakly interacting regimes at several levels of approximation, first within first-order perturbative expansions of $\alpha(\ll 1)$ [2] and later by renormalization-group (RG) calculations $[2,4,8]$; their broad consensus is that a logarithmic correction on $v$ grows upon decreasing the scale of energy $E$ towards the crossing points $(E=0)$, driving a nonlinear reshaping of Dirac cones, as reported in graphene [9] and more recently in an organic material $\alpha$-(BEDT-TTF $)_{2} \mathrm{I}_{3}$ under pressure $[10,11]$.

For strong interaction (typically $\alpha>1$ ), a remarkable breakdown of the massless-fermion picture has been suggested at charge neutrality in honeycomb lattice [12-25] as well as in Weyl semimetals [26-36]; following various analyses within Monte Carlo, RG, and mean-field techniques, this breakdown is ascribed to an excitonic condensation of electron-hole (el-h) pairs by the attractive Coulomb interaction, involving occupied states in the conduction band and empty states in the valence band near the crossing points [3,4,37-40]. Notably, the transition lifts the degeneracy of the conical dispersion associated with a pseudospin-1/2 degree of freedom. The projection of this degree onto the momentum is known as chirality $[4,41]$. Here, we focus on this pairing instability especially in two-dimensional (2D) nodal semimetals, and dub it as "chiral excitonic instability" to recall its link to the chirality (which is a solid-state equivalent of the chiral symmetry breaking in high-energy physics [41]). 
(a)

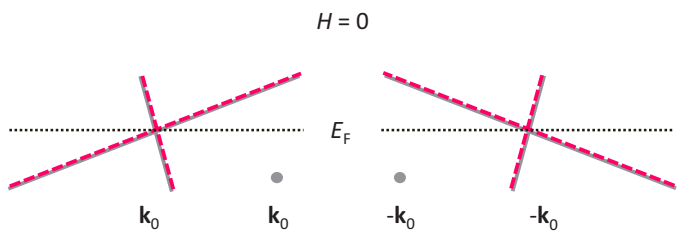

(c)

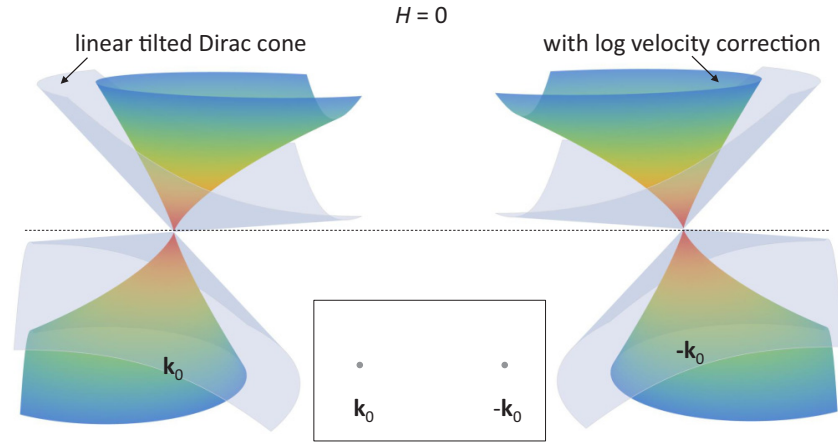

(b)

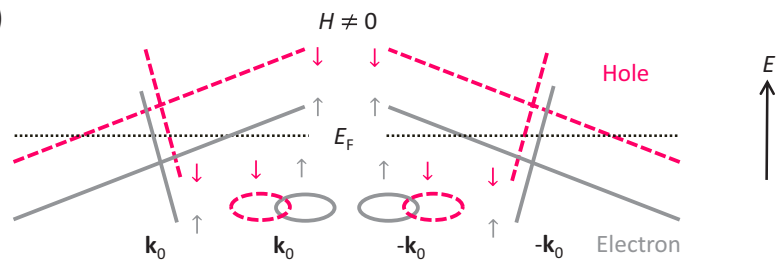

(d)

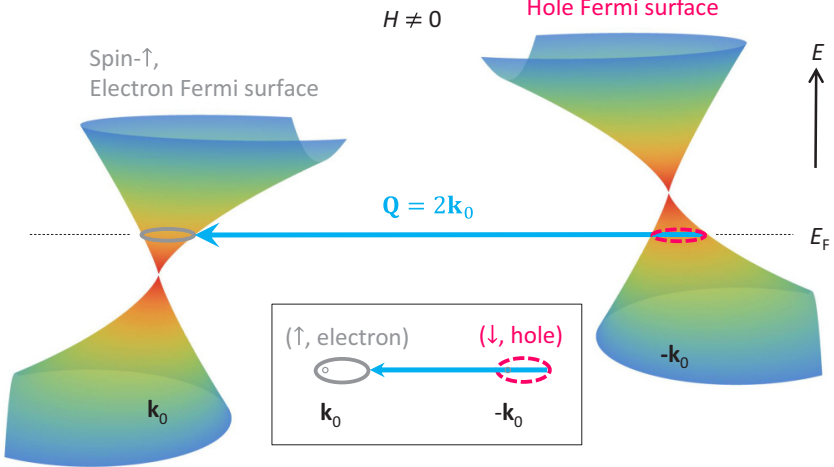

FIG. 1. Intervalley Fermi-surface nesting relevant to chiral excitonic instability in 2D tilted Dirac cones at charge neutrality. (a), (b) Spin splitting of tilted cones. Application of an in-plane magnetic field $H$ removes the spin degeneracy, and generates elliptic Fermi pockets for the spin- $\uparrow$ electrons and the spin- $\downarrow$ holes. (c), (d) The corresponding splitting of the tilted cones in $\alpha$-(BEDT-TTF) $)_{2} \mathrm{I}_{3}$. The linear dispersion (outer transparent cones) and the logarithmically reshaped dispersion (inner colored cones) are highlighted. The self-energy effects by the Coulomb interaction are incorporated [11] (calculated by the RG approach using the bare coupling of $\alpha=12.6$; see Sec. II B). For clarity, the cones for the spin- $\uparrow$ electrons (left; $\mathbf{k}_{0}$ ) and the spin- $\downarrow$ holes (right; $-\mathbf{k}_{0}$ ) are selectively depicted in (d). Inset of (c) and (d): Fermi surfaces at $H=0$ (c) and $H \neq 0$ (d). The arrow represents perfect el-h Fermi-surface nesting in intervalley excitation process, with the nesting vector of $\mathbf{Q}=2 \mathbf{k}_{0}$.

For the 2D Dirac cones in general, a pair of Dirac points (or valleys) appears in the first Brillouin zone at incommensurate wave number vectors $\pm \mathbf{k}_{0}$, and is protected by space and time inversion symmetries $[42,43]$. At charge neutrality, chiral excitonic instability can therefore develop around these points either within a cone (i.e., intravalley) or in a path connecting two different cones (intervalley). The electron spin is degenerate, and thus an applied in-plane magnetic field $H$ causes Zeeman splitting of the cones, which shifts the spin- $\uparrow$ cone from the spin- $\downarrow$ cone in each valley and generates both electron and hole Fermi pockets. Aleiner et al. [44] have discussed the influence of this Zeeman effect on the instability for graphene based on a weak-coupling mean-field treatment of gap equations, and have highlighted the importance of interband el-h Fermi-surface nesting, involving spin- $\uparrow$ electrons and spin- $\downarrow$ holes in the different bands. In graphene, the cones are isotropic and vertical with their crossing points locating at the corners of the first Brillouin zone, which leads the pockets to have identical circular shapes in each valley. Consequently, perfect el-h nesting is realized for both intravalley pairings and intervalley pairings, allowing the instability to equally grow for both cases.

On the contrary, the situation can be quite distinctive when the cones are anisotropic and/or have a tilted axis. The quasi-2D electron system in the organic conductor $\alpha$-(BEDT-TTF $)_{2} \mathrm{I}_{3}$ [where BEDT-TTF is bis(ethylenedithio)tetrathiafulvalene] provides a good example of this, where extensive studies have revealed the presence of a pair of 2D tilted Dirac cones under hydrostatic pressure $[10,11,45-53]$ that are charge neutral by $3 / 4$ filling of the electronic band [54-61]. In this system, the cones are isotropic but canted towards each valley [Fig. 1(a)], which in an in-plane $H$ lifts the spin degeneracy and generates elliptic Fermi pockets for the electron and hole bands near the crossing points (at $\pm \mathbf{k}_{0}$ ), where the electron pocket is relatively shifted from the hole pocket in opposite directions at the two valleys [Fig. 1(b)]. Then, there is poor interband el-h Fermisurface nesting between the spin- $\uparrow$ and spin- $\downarrow$ pockets for intravalley pairings, whereas perfect el-h nesting is realized for intervalley pairings [inset of Fig. 1(d)]. Therefore, one would expect that chiral excitonic instability may selectively grow for the latter process due to better nesting; in fact, this point is supported by a recent ${ }^{13} \mathrm{C}$ nuclear magnetic resonance (NMR) study at low temperature $(T)$, which finds upon cooling (besides the cone reshaping $[10,62]$ ) an anomalous upturn in the spin-lattice relaxation rate $1 / T_{1}$ that is numerically accounted for by precursor spin-triplet (transverse) fluctuations growing prior to an intervalley excitonic condensate at charge neutrality [11] [characterized by a nesting vector $\mathbf{Q}=2 \mathbf{k}_{0}$; see Figs. 1(c) and 1(d)].

If nesting proves to be pivotal to the pairings, one would expect a large impact of $H$ variations on the instability since 
an increased $H$ enlarges the sizes of Fermi pockets in each valley, and may thereby provide a larger gain in condensation energy by enhanced nesting. By contrast, a small shift of the chemical potential $\mu$ off the neutrality point considerably reduces el-h symmetry, and may thus weaken the pairing instability by degraded el-h nesting. In particular, the latter influence seems to be significant in $\alpha$-(BEDT-TTF $)_{2} \mathrm{I}_{3}$ under pressure because previous Hall measurements reported a sign change in low- $T$ Hall coefficient in some samples [63], which is ascribed by a model calculation [64] to small el-h asymmetry and a tiny electron self-doping effect of a size of a few ppm of the conduction band. Note that thermopower experiments are in line with this suggestion $[53,65]$. To construct a concrete theoretical picture within the excitonic framework, it is therefore very important to quantitatively assess the impacts of these $H$ - and $\mu$-variation effects on the intervalley paring instability, and check their influence on the precursor spin-transvers fluctuations seen by $1 / T_{1}$. However, the pairing instability has not yet been studied as functions of these parameters for tilted cones, and the relation between the transport and ${ }^{13} \mathrm{C}$-NMR data in pressurized $\alpha$-(BEDT-TTF $)_{2} \mathrm{I}_{3}$ remains largely unclear.

Here, to better understand the pairing instability in $2 \mathrm{D}$ tilted cones, we extend our previous theoretical analyses at charge neutrality in Ref. [11], and specifically investigate the impacts of variations in $\mu$ and $H$ on intervalley chiral excitonic condensate and its associated spin fluctuations. To this end, we use the tilted cones in pressurized $\alpha$-(BEDT-TTF $)_{2} \mathrm{I}_{3}$ as our testing model, whereas the argument is not restricted to this material but can be extended to generic massless cones. We assume a continuum model for the massless Dirac fermions in this material, and consider the Coulomb interaction by focusing on a ladder vertex of transverse spin susceptibility and self-energy $\Sigma$ corrections in a RG approach (incorporating both the momentum and frequency dependence [66] of $\Sigma$ ). The band parameters are taken from those reported by the previous fits to the ${ }^{13} \mathrm{C}$-Knight shift data [10], which rely on high-pressure parameters analyzed by Katayama et al. [57] based on a first-principle study of Kino et al. [54]. We restrict our attention to the vicinity of the charge-neutrality point, and numerically evaluate the instability via studying a $T-\mu-H$ phase diagram using a weak-coupling mean-field theory. In relation to this diagram, we then calculate the corresponding precursor spin-transverse fluctuations and check their influence on the $T$ dependence of $1 / T_{1}$, as functions of $\mu$ and $H$. We discuss these results with additionally performed ${ }^{13} \mathrm{C}-1 / T_{1}$ measurements in pressurized $\alpha$-(BEDT-TTF $)_{2} \mathrm{I}_{3}$, which jointly reveals that the transport-suggested tiny $\mu$ shift in this system can sensitively suppress the excitonic spin fluctuations, in accord with the intervalley Fermi-surface nesting scenario.

The paper is organized as follows. In Sec. II, we review the tilted Weyl model of pressurized $\alpha$-(BEDT-TTF $)_{2} \mathrm{I}_{3}$, discuss the expressions of transverse spin susceptibility (linked to $1 / T_{1}$ ), and comment on the treatment of the interaction ladder vertex. The methods and approximations involved in quantifying realistic contributions from the Coulomb interaction to the self-energy $\Sigma$ within the RG approach as well as deriving a weak-coupling mean-field gap equation are briefly described. Section III discusses numerical results together with supportive experimental data of ${ }^{13} \mathrm{C}-1 / T_{1}$ measurements in pressurized $\alpha$-(BEDT-TTF $)_{2} \mathrm{I}_{3}$. We provide a summary and discuss possible relevance to generic semimetals in Sec. IV. Details of calculations and supportive experiments are given in the Supplemental Material [67].

\section{CALCULATION}

\section{A. Continuum model for electrons in $\alpha$-(BEDT-TTF $)_{2} I$}

The noninteracting quasi-2D electrons in the pressurized organic conductor $\alpha$-(BEDT-TTF $)_{2} \mathrm{I}_{3}$ having valleys with a tilted dispersion relation are described in an $8 \times 8$ matrix formula (by a generalized Weyl model called the tilted Weyl Hamiltonian) as $[10,11]$

$$
\begin{aligned}
H_{0}= & \hbar\left(\mathbf{w} \cdot \mathbf{k} \hat{\sigma}_{0} \otimes \hat{\tau}_{z}+v_{x} k_{x} \hat{\sigma}_{x} \otimes \hat{\tau}_{z}+v_{y} k_{y} \hat{\sigma}_{y} \otimes \hat{\tau}_{0}\right) \otimes \hat{s}_{0} \\
& -\mu-\frac{g}{2} \mu_{B} H \hat{\sigma}_{0} \otimes \hat{\tau}_{0} \otimes \hat{s}_{z},
\end{aligned}
$$

where the chemical potential $\mu$ and the electron Zeeman term are explicitly included, with the electron $g$ factor of the size of $g=2$ [68]. The velocities $\mathbf{w}=\left(w_{x}, w_{y}\right)$ and $\mathbf{v}=\left(v_{x}, v_{y}\right)$ describe the tilt and the anisotropy of the Dirac cone, respectively. The total Hamiltonian considering the long-range part of the Coulomb interaction is given by

$$
H_{\text {eff }}=\sum_{\mathbf{k}} \Psi_{\mathbf{k}}^{\dagger} H_{0} \Psi_{\mathbf{k}}+\sum_{\mathbf{q}} V_{0}(\mathbf{q}) \rho(\mathbf{q}) \rho(-\mathbf{q})
$$

in terms of an eight-component creation operator $\Psi_{\mathbf{k}}^{\dagger}=c_{\mathbf{k}, v, s, \eta}^{\dagger}$, the density operator $\rho(\mathbf{q})=\sum_{\mathbf{k}} \sum_{\nu, s, \eta}$ $c_{\mathbf{k}, v, s, \eta}^{\dagger} c_{\mathbf{k}+\mathbf{q}, v, s, \eta}$, and the Fourier transform of the Coulomb potential $V_{0}(\mathbf{q})=2 \pi e^{2} / \varepsilon|\mathbf{q}|$, where $\mathbf{q}$ and $\mathbf{k}=\left(k_{x}, k_{y}\right)$ are 2D wave number vectors defined only around the band-crossing points at $\pm \mathbf{k}_{0}$. (We omit backscattering and Umklapp processes such that both $\mathbf{k}$ and $\mathbf{k}+\mathbf{q}$ are restricted around these degeneracy points; i.e., $\mathbf{q} \ll 2 \mathbf{k}_{0}$.)

Here, the index $\eta=1 / R(-1 / L)$ stands for the valley at $\mathbf{k}_{0}$ $\left(-\mathbf{k}_{0}\right)$, and $s=1 / \uparrow(-1 / \downarrow)$ corresponds to the up (down) spin projection. The creation (annihilation) operator $c_{\mathbf{k}, v, s, \eta}^{\dagger}$ $\left(c_{\mathbf{k}, \nu, s, \eta}\right)$ is based on the Luttinger-Kohn (LK) representation [69], described using the Bloch's functions at $\pm \mathbf{k}_{0}$ as the basis of the wave functions $[56,70]$. The index $v=1 / a(-1 / b)$ then denotes the two bases in the LK representation, and we use a notation $\bar{v}=-v$. The corresponding orbitals in this representation are given in Ref. [67]. The three matrices $\hat{\sigma}_{i}, \hat{\tau}_{i}$, and $\hat{s}_{i}$ stand for the Pauli matrices that represent LK pseudospin $1 / 2$, valley pseudospin $1 / 2$, and real spin $1 / 2$, respectively, with the three indices taking one of the four possible values $(i, j=0, x, y$, and $z)$. The index 0 represents a unit matrix.

Throughout this paper, we omit the $T$ dependence of $\mu$. This approximation is justified as the low- $T$ Hall measurements and associated theories [63,64] find only a negligibly small variation of $\mu(T)$, especially in the $T$ range of our interest (i.e., $T<10 \mathrm{~K}$ ).

\section{B. Self-energy corrections}

The self-energy-induced renormalization of velocity by the long-range part of the Coulomb interaction is considered at the level of one-loop RG calculations in leading order in $1 / N$ 
$(N \gg 1)$ as discussed previously [10,11], which is valid both for weak and strong Coulomb interaction, where $N=4$ is the number of fermion species standing for two spin projections and two valleys $[2,4,8]$. Briefly, we start from a tilted dispersion relation

$$
E_{ \pm}(\mathbf{k})=\hbar\left(\mathbf{w} \cdot \mathbf{k} \pm \sqrt{v_{x}^{2} k_{x}^{2}+v_{y}^{2} k_{y}^{2}}\right)
$$

and combine it with one-loop level RG flow equations of $\mathbf{v}=$ $\left(v_{x}, v_{y}\right)[10]$,

$$
\begin{aligned}
& \frac{1}{v_{x}} \frac{d v_{x}}{d l}=\frac{8}{\pi^{2} N} \int_{0}^{2 \pi} \frac{d \varphi}{2 \pi} 2 \cos ^{2} \varphi F\left(g_{\varphi}\right), \\
& \frac{1}{v_{y}} \frac{d v_{y}}{d l}=\frac{8}{\pi^{2} N} \int_{0}^{2 \pi} \frac{d \varphi}{2 \pi} 2 \sin ^{2} \varphi F\left(g_{\varphi}\right) .
\end{aligned}
$$

Here, $\mathbf{k} /|\mathbf{k}|=(\cos \varphi, \sin \varphi)$ is measured from $\mathbf{k}_{0}, l=$ $\ln (\Lambda / k)$ (with $|\mathbf{k}|=k$ ) is a momentum scale measured in the unit of a momentum cutoff $\Lambda=0.667 \AA^{-1}$ of the size of an inverse lattice constant [71] and circular around the Dirac point, and $F\left(g_{\varphi}\right)$ is a function of the form $F\left(g_{\varphi}\right)=\left(-\pi / 2+g_{\varphi}+\arccos g_{\varphi} / \sqrt{1-g_{\varphi}^{2}}\right) / g_{\varphi}$ with $g_{\varphi}=2 \pi e^{2} N /\left(16 \varepsilon \sqrt{v_{x}^{2} \sin ^{2} \varphi+v_{y}^{2} \cos ^{2} \varphi}\right)$. The bare coupling constant of the Coulomb interaction is given by $\alpha=$ $e^{2} /\left(4 \pi \varepsilon_{0} \varepsilon \hbar \sqrt{\left.v_{x}^{2} \sin ^{2} \varphi+v_{y}^{2} \cos ^{2} \varphi\right)}\right.$ [10], which is approximated to be $\alpha \approx e^{2} / 4 \pi \varepsilon_{0} \varepsilon \hbar v_{0}$ since the anisotropy is negligibly small in $\alpha$-(BEDT-TTF $)_{2} \mathrm{I}_{3}\left(v_{x} \approx v_{y} \equiv v_{0}\right)$ [57]. Reflecting the RG flow, $\mathbf{v}$ grows logarithmically as a function of $\Lambda / k$, whereas $\mathbf{w}$ does not flow at oneloop level. Notice that Eq. (4) considers screening effect of the Coulomb interaction including polarization bubbles in the self-energy [wavy lines in Fig. S1(b) of the Supplemental Material [67]].

To be specific, we will incorporate the RG flow of Eq. (4) obtained from fits to the ${ }^{13} \mathrm{C}$-Knight shift data in pressurized $\alpha$-(BEDT-TTF $)_{2} \mathrm{I}_{3}$ by Hirata et al. [10], using an effective tight-binding (TB) model in Ref. [57] as a minimal starting point. To adjust the initial velocity values at the cutoff $(|\mathbf{k}|=\Lambda)$, a phenomenological parameter $u$ is introduced as $\mathbf{v}=u \mathbf{v}^{\mathrm{TB}}$ and $\mathbf{w}=u \mathbf{w}^{\mathrm{TB}}$, where $\mathbf{v}^{\mathrm{TB}}$ and $\mathbf{w}^{\mathrm{TB}}$ are the velocity values derived from the effective TB model [57]. Optimizing the two parameters $\varepsilon$ and $u$ by least-square fits, we get $(\varepsilon, u) \approx(30,0.35)$, which yields $\alpha=12.6$ at the high-energy cutoff (at $|\mathbf{k}| / \Lambda=1$ ) using $v_{0}=u v^{\mathrm{TB}}$ (where
$v^{\mathrm{TB}}=2.4 \times 10^{4} \mathrm{~m} \mathrm{~s}^{-1}$ is the corresponding velocity in the gentle slope of the tilted cone) [57], and an effective coupling of the order of unity at low energy near the crossing points (at $|\mathbf{k}| / \Lambda \ll 1$ ). (For the detail of the RG flow, see the Supplemental Information of Ref. [11].) Notice that we have $u<1$ from the fits, which signals a reduced electronic bandwidth as has been often discussed in correlated electron materials, and ascribed to frequency dependence of the self-energy due to the short-range part of the Coulomb interaction [66].

The parameters obtained in this way thus incorporate both the logarithmic velocity flow and the bandwidth suppression. Throughout this study, we will use these as phenomenological but quantitative estimates of self-energy effects [i.e., the reshaped cones in Figs. 1(c) and 1(d)].

\section{Transverse excitonic spin fluctuations}

We assess the influence of the Coulomb interaction on transverse excitonic spin fluctuations in a ladder-type approximation. In particular, we identify the impacts of variations in both chemical potential $\mu$ near the crossing points and in-plane magnetic field $H$ on the nuclear-spin-lattice relaxation rate $1 / T_{1}$, linked to a wave number average of the imaginary part of the transverse dynamic spin susceptibility, $\sum_{\mathbf{Q}} \operatorname{Im} \chi_{\perp}(\mathbf{Q}, \omega)$, where $\mathbf{Q}$ is a wave number vector and $\omega$ is a frequency in megahertz region [72]. [The full expression of $1 / T_{1}$ is given in Eq. (S8) of the Supplemental Material [67].] To this end, it is sufficient to focus on intervalley excitonic instability for even-parity, spin-triplet (or spin-transverse) pairings as a first approximation, which is shown to give major contributions in $\alpha$-(BEDT-TTF $)_{2} \mathrm{I}_{3}$ at charge neutrality [11]. (See the Supplemental Material for the corresponding order parameter [67].) Hereafter, we will assume a pair of gapless points placed along the $k_{x}$ axis at $k_{x}=0$ and $k_{x}=2 k_{0}$ for the sake of clarity.

We use a generalized expression of $\chi_{\perp}(\mathbf{Q}, \omega)$ that involves the two bases in the LK representation $(v=a, b)$ and picks up intervalley el-h excitation process (connecting $\eta=R$ and $L$ with a momentum transfer $2 \mathbf{k}_{0} \hbar$ ). The corresponding intervalley susceptibility is obtained by an analytic continuation of

$$
\begin{aligned}
\chi_{\perp}^{R, L}\left(\mathbf{q}, i \omega_{m}\right)= & \sum_{\mathbf{k}, v}\left[\mathcal{M}_{\nu \nu \bar{v} \bar{v}}^{R, L}\left\{\Lambda_{+}^{v}\left(\mathbf{k} ; \mathbf{q}, i \omega_{m}\right) \chi_{+}^{v}\left(\mathbf{k} ; \mathbf{q}, i \omega_{m}\right)+\Lambda_{-}^{v}\left(\mathbf{k} ; \mathbf{q}, i \omega_{m}\right) \chi_{-}^{v}\left(\mathbf{k} ; \mathbf{q}, i \omega_{m}\right)\right\}\right. \\
& \left.+\mathcal{M}_{\nu \bar{\nu} \nu \bar{v}}^{R, L}\left\{\Lambda_{-}^{v}\left(\mathbf{k} ; \mathbf{q}, i \omega_{m}\right) \chi_{+}^{v}\left(\mathbf{k} ; \mathbf{q}, i \omega_{m}\right)+\Lambda_{+}^{v}\left(\mathbf{k} ; \mathbf{q}, i \omega_{m}\right) \chi_{-}^{v}\left(\mathbf{k} ; \mathbf{q}, i \omega_{m}\right)\right\}\right]
\end{aligned}
$$

where $\varepsilon_{n} \quad\left(\omega_{m}\right)$ is the fermionic (bosonic) Matsubara frequency, $\mathcal{M}_{v_{1} \nu_{2} v_{3} v_{4}}^{\eta, \eta^{\prime}}$ is a constant form factor associated with the tilt of the valleys, $\Lambda_{ \pm}^{v}\left(\mathbf{k} ; \mathbf{q}, i \omega_{m}\right)$ are vertex contributions of a ladder type, and $\chi_{ \pm}^{v}\left(\mathbf{k} ; \mathbf{q}, i \omega_{m}\right)$ are irreducible susceptibilities given by

$$
\begin{aligned}
\chi_{+}^{\nu}\left(\mathbf{k} ; \mathbf{q}, i \omega_{m}\right)= & -T \sum_{\varepsilon_{n}} G_{\uparrow, R}^{\nu \nu}\left(\mathbf{k}+\mathbf{q}, i \varepsilon_{n}+i \omega_{m}\right) \\
& \times G_{\downarrow, L}^{\bar{v} \bar{v}}\left(\mathbf{k}, i \varepsilon_{n}\right),
\end{aligned}
$$




$$
\begin{aligned}
\chi_{-}^{\nu}\left(\mathbf{k} ; \mathbf{q}, i \omega_{m}\right)= & -T \sum_{\varepsilon_{n}} G_{\uparrow, R}^{\nu \bar{v}}\left(\mathbf{k}+\mathbf{q}, i \varepsilon_{n}+i \omega_{m}\right) \\
& \times G_{\downarrow, L}^{\nu \bar{\nu}}\left(\mathbf{k}, i \varepsilon_{n}\right) .
\end{aligned}
$$

Here, $\hat{G}_{s, \eta}=\left[G_{s, \eta}^{\nu \nu^{\prime}}\right]$ is a Green's function for 2D massless Dirac fermions with a tilted dispersion relation, which has a matrix structure based on the LK representation. Note that the aforementioned self-energy effects are incorporated in $\hat{G}_{s, \eta}$. The corresponding form factor $\mathcal{M}_{v_{1} v_{2} v_{3} v_{4}}^{\eta, \eta^{\prime}}$ and the Green's function $\hat{G}_{s, \eta}$ are defined in the Supplemental Material [67]. Using these irreducible susceptibilities in Eqs. (6) and (7), the vertex contributions are expressed by a Bethe-Salpeter-type equation [73-75],

$$
\begin{aligned}
\Lambda_{ \pm}^{v}\left(\mathbf{k} ; \mathbf{q}, i \omega_{m}\right)= & 1+\sum_{\mathbf{k}^{\prime}} V_{0}\left(\mathbf{k}-\mathbf{k}^{\prime}\right)\left[\Lambda_{ \pm}^{v}\left(\mathbf{k}^{\prime} ; \mathbf{q}, i \omega_{m}\right)\right. \\
& \times \chi_{+}^{v}\left(\mathbf{k}^{\prime} ; \mathbf{q}, i \omega_{m}\right)+\Lambda_{\mp}^{v}\left(\mathbf{k}^{\prime} ; \mathbf{q}, i \omega_{m}\right) \\
& \left.\times \chi_{-}^{v}\left(\mathbf{k}^{\prime} ; \mathbf{q}, i \omega_{m}\right)\right] .
\end{aligned}
$$

The corresponding diagrams are given in Fig. S1 of the Supplemental Material [67], and we will convert the analytic continuation of the susceptibility in Eq. (5) to the relaxation rate divided by $T, 1 / T_{1} T$, by taking a wave number average of its imaginary part as in Eq. (S8) of the Supplemental Material [67].

At the level of random phase approximation, the $\mathbf{k}$ dependence in Eq. (8) can be separated from the dependence on $\mathbf{q}$ and $\omega_{m}$ as $\Lambda_{ \pm}^{v}\left(\mathbf{k} ; \mathbf{q}, i \omega_{m}\right) \simeq \Delta_{\mathbf{k}} \Lambda_{ \pm}^{v}\left(\mathbf{q}, i \omega_{m}\right)$, which yields

$$
\Lambda_{ \pm}^{v}\left(\mathbf{q}, i \omega_{m}\right) \simeq\left[1-\lambda\left(\mathbf{q}, i \omega_{m}\right)\right]^{-1}
$$

and a linearized weak-coupling gap equation

$$
\begin{aligned}
\lambda\left(\mathbf{q}, i \omega_{m}\right) \Delta_{\mathbf{k}}= & \sum_{\mathbf{k}^{\prime} v} V_{0}\left(\mathbf{k}-\mathbf{k}^{\prime}\right) \Delta_{\mathbf{k}^{\prime}}\left[\chi_{+}^{v}\left(\mathbf{k}^{\prime} ; \mathbf{q}, i \omega_{m}\right)\right. \\
& \left.+\chi_{-}^{v}\left(\mathbf{k}^{\prime} ; \boldsymbol{q}, i \omega_{m}\right)\right],
\end{aligned}
$$

where $\Delta_{\mathbf{k}}$ is a gap and $\lambda\left(\mathbf{q}, i \omega_{m}\right)$ is a corresponding eigenvalue. We will numerically evaluate this self-consistent equation in Eq. (10) at mean-field level, and use $\lambda(0,0) \equiv \lambda$ to assess excitonic instability, which favors a gap opening at the crossing points when $\lambda=1$ is reached.

To be compatible with the RG flow in Sec. II B [cf. Eq. (4)], we use a low-energy effective value of $\alpha_{\text {eff }}=1$ as our approximate constant Coulomb coupling in Eqs. (5)-(10). This approximation is justified at low temperature $(T<10$ $\mathrm{K}$ ), where the RG flow of the coupling appears to be saturated and the coupling becomes of the order of unity in $\alpha$-(BEDT-TTF $)_{2} \mathrm{I}_{3}$ (see Ref. [11]).

Notice that we will omit intravalley el-h pairings as they barely contribute to chiral excitonic instability even at charge neutrality. The reason is not only due to poor intravalley el-h nesting in tilted valleys as mentioned in Sec. I [cf. Fig. 1(b)] [11], but also because of chiral property of massless Dirac fermions, which suppresses backscattering processes for the intravalley pairings but not for the intervalley pairings [76,77]. Constructing models relying solely on intervalley contributions thus provides a reliable starting point for dis- cussing chiral excitonic instability of 2D tilted Dirac cones. [A relevant chirality factor is implicitly involved in Eqs. (6) and (7).]

Similarly, spin-singlet (spin-longitudinal) instability will be neglected, which is shown to be suppressed upon increasing in-plane magnetic field at charge neutrality (see Ref. [11]).

\section{RESULTS AND DISCUSSION}

Chiral excitonic instability and its impact on the spinlattice relaxation rate in pressurized $\alpha$-(BEDT-TTF $)_{2} \mathrm{I}_{3}$ with small charge off-neutrality $(\mu \neq 0)$ at in-plane magnetic field $H$ is determined by two logarithmically reshaped massless Dirac cones for even-parity, spin-triplet (transverse), and intervalley pairings. (Notice that this is a natural extension of Ref. [11], performed at $\mu=0$ and small $H$, to a more general case.) At a weak-coupling mean-field-level treatment of gap equation, we find that the contribution from the two cones to intervalley excitonic response strongly depends on $\mu$ and $H$ (Sec. III A). The way this contribution affects the precursor transverse spin fluctuations is also highly dependent on these parameters (Sec. III B). The additional experimental data of the ${ }^{13} \mathrm{C}$-spin-lattice relaxation rate in pressurized $\alpha$-(BEDT-TTF $)_{2} \mathrm{I}_{3}$ show a good qualitative agreement with this prediction, filling the gap between ${ }^{13} \mathrm{C}-\mathrm{NMR}$ study at $\mu=0$ [11] and transport results indicating $\mu \neq 0[53,63-65]$ in this material (Sec. III C).

\section{A. Mean-field phase diagram for the intervalley response}

To highlight the intervalley-nesting dependence in chiral excitonic instability, we first focus on a mean-field critical temperature $T_{\mathrm{C}}$ where the gap starts to open [i.e., when $\lambda=1$ is fulfilled in Eq. (10)], and consider its explicit dependence on chemical potential and in-plane magnetic field. This analysis of a mean-field phase diagram helps us to understand the impact of these parameters on the $T$ dependence of the relaxation rate, discussed in Sec. III B.

Given that the transverse susceptibility in Eq. (5) and the corresponding gap equation in Eq. (10) pick up the intervalley el-h excitations involving spin- $\uparrow$ electrons and spin- $\downarrow$ holes, the shapes of the spin-split Fermi pockets induced by in-plane magnetic field must play an important role.

Figures 2(a) and 2(b) present schematic illustrations of the field-induced pockets around the two valleys (near $\pm \mathbf{k}_{0}$ ) at charge neutrality [Fig. 2(a)] and off-neutrality [Fig. 2(b)]. The Fermi pockets for the spin- $\uparrow$ electrons (filled lines) and the spin- $\downarrow$ holes (dashed lines) are depicted.

Intervalley el-h excitations must occur between an el pocket in one valley and a hole pocket in the other. At charge neutrality, there is perfect nesting in the intervalley pairing process with a momentum transfer of $\hbar \mathbf{Q}=2 \hbar \mathbf{k}_{0}$ [indicated by an arrow in Fig. 2(a)]. With increasing the field, the size of these pockets increases, which nevertheless keeps the perfect nesting condition intact. Consequently, the number of el-h pairs involved in nesting increases towards higher field. For off-neutral case, by contrast, el-h asymmetry causes unequally sized electron pockets and hole pockets [Fig. 2(b)]. The corresponding intervalley nesting condition is thus worsened 
(a)

Charge neutral

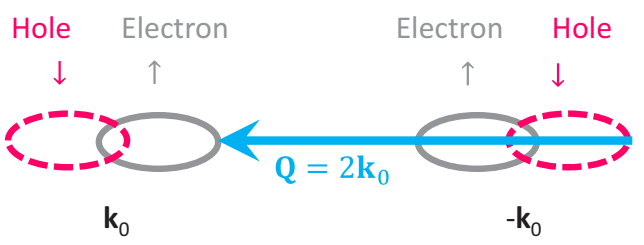

(b)

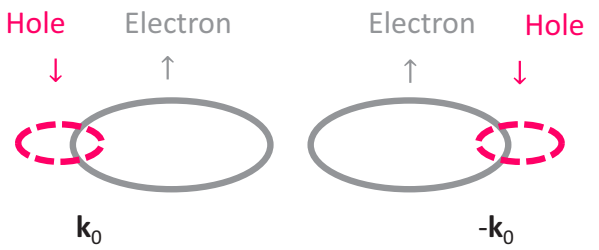

(c)

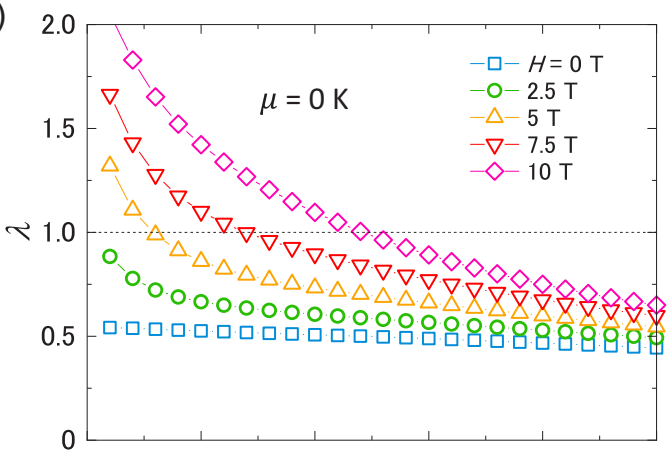

(d)

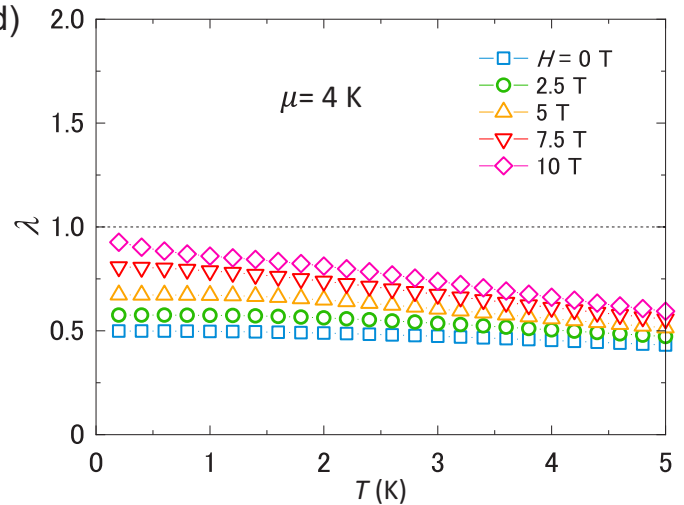

FIG. 2. Eigenvalues of intervalley chiral excitonic instability for 2D tilted Dirac cones at various doping and in-plane magnetic fields. (a), (b) Illustrations of spin-split Fermi pockets in an in-plane magnetic field $H$ for the spin- $\uparrow$ electrons (solid) and the spin- $\downarrow$ holes (dashed) at charge neutrality (a) and off-neutrality (b). Perfect interband (electron-hole) nesting in the intervalley excitation process is depicted by an arrow in (a), with the nesting vector of $\mathbf{Q}=2 \mathbf{k}_{0}$. (c), (d) Temperature dependence of the mean-field eigenvalue $\lambda$ for the even-parity, spin-triplet (transverse) instability in the intervalley pairing $\left(\mathbf{Q}=2 \mathbf{k}_{0}\right)$. Calculated $\lambda$ are shown for various in-plane magnetic field at charge neutrality $(\mu=0 \mathrm{~K})(\mathrm{c})$ and off-neutrality $(\mu=4 \mathrm{~K})(\mathrm{d})$. The effective Coulomb coupling of $\alpha_{\mathrm{eff}}=1$ is used, and the self-energy corrections are considered (see Sec. II).

compared to the neutral case, prohibiting el-h pair formation in this process.

These considerations should naturally affect the intervalley excitonic response since it is directly related to the number of el-h pairs involved in the corresponding excitation process.

Figures 2(c) and 2(d) show the $T$ dependence of the meanfield eigenvalue $\lambda$ in Eq. (10) for selected values of in-plane magnetic field at charge neutrality $(\mu=0 \mathrm{~K})$ and with small electron doping $(\mu=4 \mathrm{~K})$, respectively, where we have also plotted a line corresponding to $\lambda=1$ (dashed line). The crossing points of this line with the data define $T_{\mathrm{C}}$. As shown in Fig. 2(c), excitonic condensation (corresponding to the region with $\lambda>1$ ) is present at low temperature at charge neutrality, whose region increases to higher temperature upon raising the field. For off-neutrality, on the other hand, the eigenvalue is strongly suppressed, and there is no excitonic region in the present parameter range [Fig. 2(d)].

This situation is more clearly discernible when we look at the critical temperature as shown in Fig. 3, where we have plotted $T_{\mathrm{C}}$ as a function of temperature, chemical potential, and in-plane magnetic field. For given doping, the excitonic region (colored region in Fig. 3) increases to higher temperature with raising the field, whereas at a fixed field $T_{\mathrm{C}}$ decreases upon moving away from $\mu=0$ by doping.

This property of $T_{\mathrm{C}}$ can be directly ascribed to Fermisurface nesting that is enhanced towards higher field and suppressed with doping. Indeed, at low field, the instability grows at $\mu=0$ only above a threshold field value $\left(H_{\mathrm{c}} \approx\right.$ $1.7 \mathrm{~T})$. This is because the low-field pockets are small, and

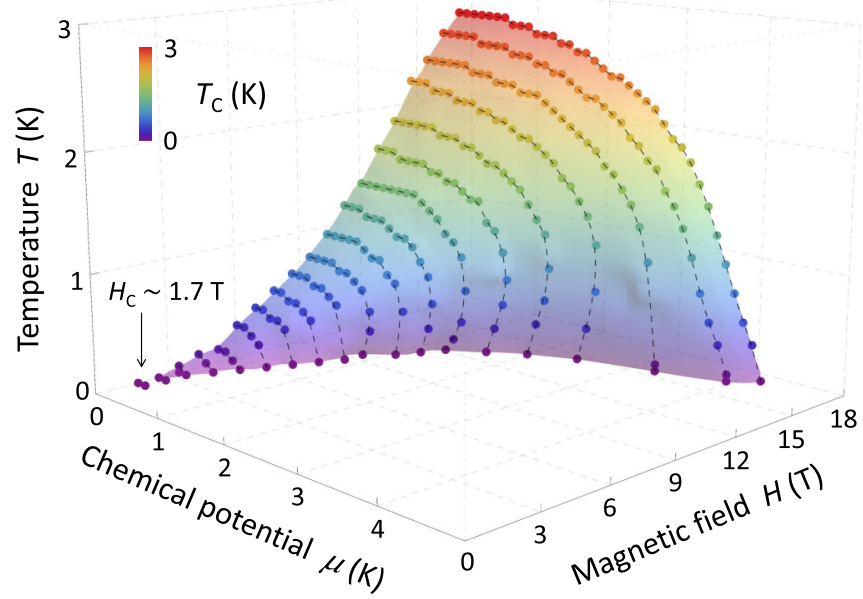

FIG. 3. Mean-field transition temperature $T_{c}$ for intervalley chiral excitonic condensate with even-parity and spin-triplet (transverse) pairings, plotted as functions of temperature $T$ (in $\mathrm{K}$ ), chemical potential $\mu$ (in $\mathrm{K}$ ), and in-plane magnetic field $H$ (in T). The physical parameters are the same as in Fig. 2. The arrow indicates the critical field $H_{c}$, and the label bar represents $T_{c}$. Dashed lines are guides to the eyes. 
(a)

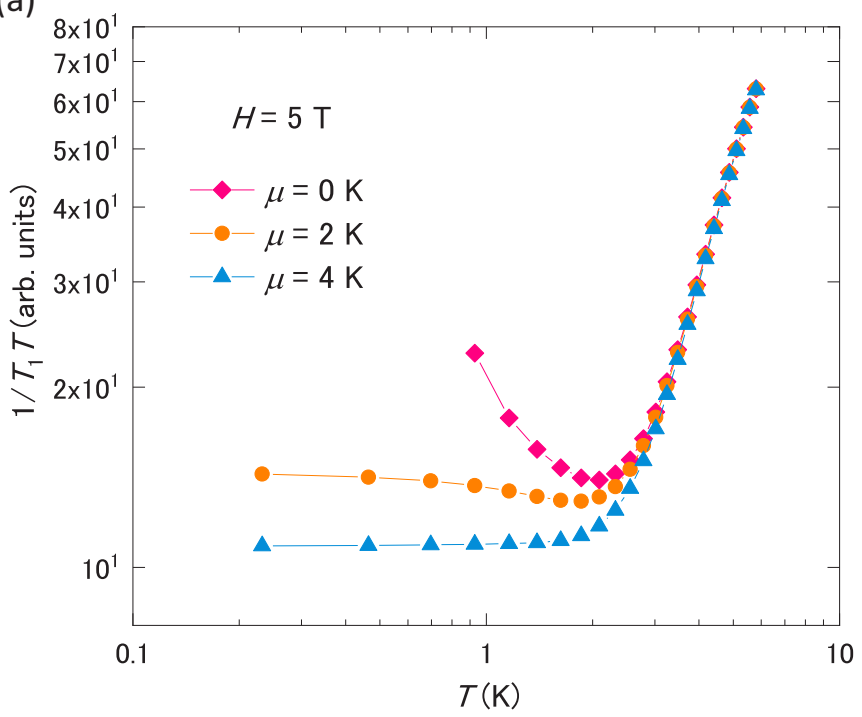

(b)

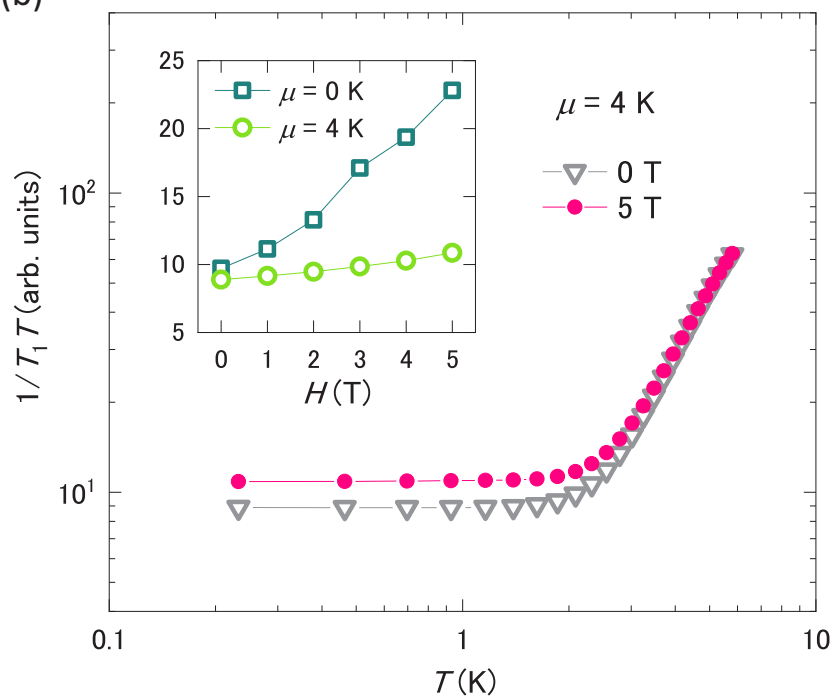

FIG. 4. Temperature dependence of the nuclear spin-lattice relaxation rate divided by temperature $1 / T_{1} T$ for various doping and in-plane magnetic fields. Panel (a) shows the case with a fixed in-plane field $(H=5 \mathrm{~T})$ and a range of small electron doping of $\mu=0,2$, and $4 \mathrm{~K}$. Panel (b) depicts the case with fixed small doping $(\mu=4 \mathrm{~K}$ ) and different fields of $H=0$ and $5 \mathrm{~T}$. Inset of panel (b) shows the field dependence at low temperature $(T=0.2 \mathrm{~K})$ at charge neutrality $(\mu=0 \mathrm{~K})$ and off-neutrality $(\mu=4 \mathrm{~K})$. The physical parameters are the same as in Figs. 2 and 3 .

accordingly the number of el-h pairs involved in nesting is greatly limited. Near $\mu=0$ at low field, moreover, the condensation is highly sensitive to doping since a small change of $\mu$ can drastically worsen nesting. By contrast, the excitonic state is present at largely doped regions for higher field. The reason is that the field-enlarged pockets weaken the relative influence of el-h asymmetry at the Fermi energy, which improves the nesting condition and thus allows an increased number of el-h pairs to participate in condensation.

We note considerable sensitivity of this instability to a shred of doping, especially near $\mu=0$. For instance, at $5 \mathrm{~T}$, the instability vanishes for $\mu \geqslant 1 \mathrm{~K}$, corresponding to electron doping of just a few ppm of the conduction band. This remarkably small size of doping is in accord with the transport analysis in $\alpha$-(BEDT-TTF $)_{2} \mathrm{I}_{3}$, as discussed in Sec. III C.

\section{B. Spin-lattice relaxation rate $1 / T_{1}$}

The nuclear spin-lattice relaxation rate $1 / T_{1}$ allows us to study slow spin dynamics at the Fermi energy. In this section, we investigate how doping and in-plane magnetic field alter the nature of precursor excitonic spin fluctuations in $\alpha$-(BEDT-TTF $)_{2} \mathrm{I}_{3}$ near charge neutrality, particularly in view of the above nesting condition in relation to the phase diagram.

For the charge-neutral Dirac cones, the low-energy excitations and fluctuations can be decoupled into two parts by means of NMR. The first part is intravalley contribution around each crossing point (at $\pm \mathbf{k}_{0}$ ), which is probed by the uniform component $(\mathbf{Q}=0)$ of the electron spin susceptibility (or the Knight shift). The second part is intervalley contribution, which appears in the $\mathbf{Q} \approx 2 \mathbf{k}_{0}$ response of the local spin susceptibility. The relaxation rate $1 / T_{1}$ picks up a sum of these two contributions as it is proportional to a $\mathbf{Q}$ average of
$\operatorname{Im} \chi_{\perp}(\mathbf{Q}, \omega)$ [cf. Eq. (S8) [67]]. In $\alpha$-(BEDT-TTF $)_{2} \mathrm{I}_{3}$, Hirata et al. $[10,11]$ revealed at charge neutrality that the $\mathbf{Q}=0$ part is almost exclusively suppressed upon cooling by the logarithmic velocity renormalization in Eq. (4) [cf. the reshaped cones in Figs. 1(c) and $1(\mathrm{~d})$ ], such that $1 / T_{1}$ becomes considerably sensitive to the $\mathbf{Q} \approx 2 \mathbf{k}_{0}$ part at low temperature. This makes the relaxation rate appealing to the study of chiral excitonic fluctuations near charge neutrality, in particular for intervalley pairings.

Here, for later comparisons with supportive experiments (Sec. III C), we discuss two representative cases, one at a fixed field and a range of small doping [Fig. 4(a)], and the other at fixed small doping and for different magnetic fields [Fig. 4(b)]. For visualization, the rate divided by temperature, $1 / T_{1} T$, will be used.

The first case is presented in Fig. 4(a), where the temperature dependence of $1 / T_{1} T$ is shown for various small electron doping $(\mu=0,2$, and $4 \mathrm{~K})$ at $5 \mathrm{~T}$, which corresponds to the intervalley excitonic response of the eigenvalues studied in Figs. 2(c) and 2(d) (upward triangles). At charge neutrality $(\mu=0 \mathrm{~K})$, the curve shows a clear upturn with decreasing temperature due to intervalley $\left(\mathbf{Q}=2 \mathbf{k}_{0}\right)$ spin fluctuations by the ladder vertex (8) growing as a precursor to the condensation [11]. A small increase of $\mu$ rapidly suppresses the fluctuations, and at $\mu=4 \mathrm{~K}$ flattens $1 / T_{1} T$ at low temperature due to worsened intervalley nesting by doping, as discussed in Sec. III A [cf. Figs. 2(a) and 2(b)]. Notice that the levelling-off of $1 / T_{1} T$ at low temperature (for $\mu=4 \mathrm{~K}$ ) is a universal characteristic of interband el$\mathrm{h}$ excitations near charge neutrality, which appears when chiral excitonic instability is absent irrespective of the size of small doping (see discussions in Ref. [11] for chargeneutral valleys, and Fig. S3 in Ref. [67] for off-neutral valleys). 

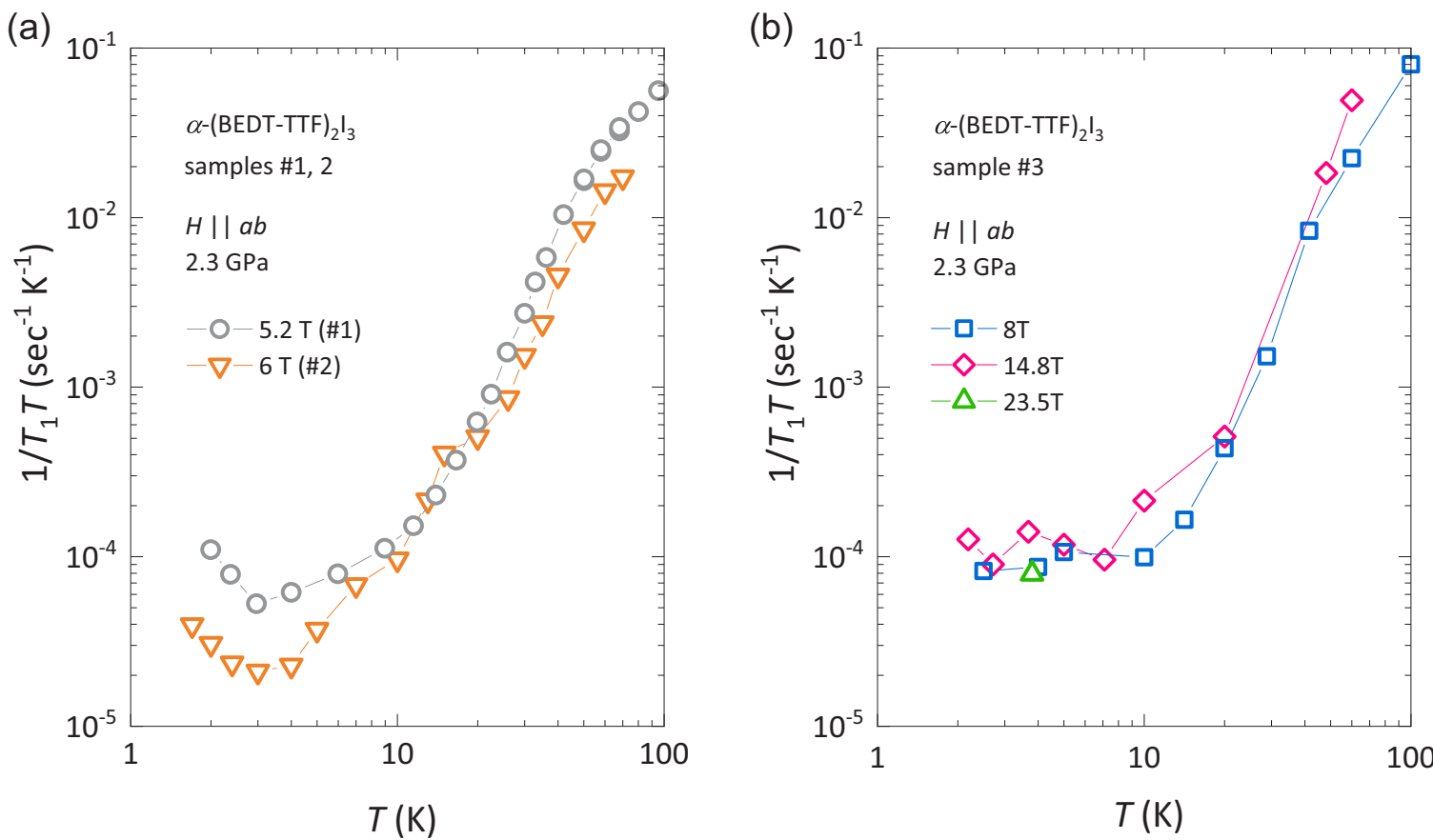

FIG. 5. Temperature dependence of $1 / T_{1} T$ in $\alpha$-(BEDT-TTF $)_{2} \mathrm{I}_{3}$ measured by ${ }^{13} \mathrm{C}-\mathrm{NMR}$ experiments in three samples (1-3) at $2.3 \mathrm{GPa}$ for various in-plane magnetic fields. Panel (a) shows the data in sample 1 (2) at a magnetic field of $H=5.2 \mathrm{~T}(6 \mathrm{~T})$ applied parallel to the crystalline $a b$ plane (2 replotted from Ref. [11]). Panel (b) depicts the corresponding results in sample 3, measured at $H=8,14.8$, and 23.5 T (for experimental details, see Ref. [67]).

In Fig. 4(b), we present $1 / T_{1} T$ for the second case, fixed small doping $(\mu=4 \mathrm{~K})$ and different magnetic fields of $H=$ 0 and $5 \mathrm{~T}$, corresponding to the response described by the eigenvalue in Fig. 2(d) (squares and upward triangles). The precursor excitonic spin fluctuations do not grow due to large el-h asymmetry and poor intervalley nesting at this doping compared to the relatively small size of $H$ [cf. Fig. 2(b)]. This is in accord with the phase diagram (Fig. 3) where these parameter regions locate far away from the excitonic dome region.

The little field dependence at off-neutrality is contrasted to the neutral case as depicted in the inset of Fig. 4(b), where we have plotted $1 / T_{1} T$ at low temperature $(T=0.2 \mathrm{~K})$ against field for $\mu=0$ and $4 \mathrm{~K}$. The relaxation rate shows a sizeable (moderate) increase with raising the field at charge neutrality (off-neutrality) as a direct consequence of larger (weaker) elevation of the low- $T$ eigenvalue towards higher field in Fig. 2(c) [Fig. 2(d)]. The larger field dependence for smaller doping in the relaxation rate agrees with the intervalleynesting picture because better nesting near charge neutrality provides stronger instability and, therefore, larger precursor fluctuations as the pocket sizes are enlarged upon increasing the field.

\section{Comparison with experiments in $\alpha$-(BEDT-TTF $)_{2} \mathbf{I}_{3}$}

To assess the qualitative validity of these calculations, we have additionally performed ${ }^{13} \mathrm{C}$-NMR experiments in pressurized $\alpha$-(BEDT-TTF $)_{2} I_{3}$ and have measured the spin-lattice relaxation rate (experimental details are given in Ref. [67]). Figures 5(a) and 5(b) present the temperature dependence of $1 / T_{1} T$ in in-plane magnetic fields, measured for three representative samples (labeled 1-3). As shown in Fig. 5(a), 1/ $T_{1} T$ in sample 1 (along with 2 replotted from Ref. [11]) decreases upon cooling and shows an abrupt upturn at low temperature (for $H=5.2$ to $6 \mathrm{~T}$ ). This can be well understood by the precursor intervalley $\left(\mathbf{Q}=2 \mathbf{k}_{0}\right)$ spin fluctuations growing prior to chiral excitonic condensate at charge neutrality, as discussed in Sec. III B [cf. Fig. 4(a) for $\mu=0$ K]. For sample 3 in Fig. 5(b), by contrast, $1 / T_{1} T$ exhibits no upturn but a levelling-off-like behavior at below $10 \mathrm{~K}$. Moreover, we find this low- $T$ flattening to have little field dependence in a range from $H=8$ to 23.5 T. These features are in accord with the above-mentioned expectation for interband el-h excitations in 2D cones when the instability is suppressed by a small shift of chemical potential of a size of just a few Kelvin (submillielectronvolt) off the charge-neutrality point [cf. Fig. 4(b)].

The contrasting observations for samples 1 (2) and 3 in Fig. 5 draw a qualitative parallel with the results in Fig. 4, testifying the validity of our calculations based on laddertype approximation. They further lend support for the idea that intervalley chiral excitonic instability in this system is highly sensitive to a small variation of $\mu$ around the crossing points, such that the precursor fluctuations are absent in a relatively doped sample (3) but present in less-doped ones ( 1 and 2). Moreover, the remarkably small doping considered above quantitatively agrees with what has been suggested by transport experiments in out-of-plane magnetic fields $[53,63,65,78]$ and a relevant calculation within a linearresponse theory [64]. It also does not conflict with more recent magnetotransport studies in an in-plane magnetic field $[51,79]$.

These results suggest that there is a possible underlying instability towards intervalley-excitonic ground state, which may be able to be observed in strong in-plane magnetic field at very low temperature if and only if the chemical potential 
is finely tuned to the crossing points. On the other hand, the fact that $\mu$ in this organic material is confined that close to the crossing points (in a submillielectronvolt range) is contrasted with monolayer graphene-which usually suffers from corrugated structures (called ripples) and potential inhomogeneity (known as charge puddles) [80] that make fine tuning of $\mu$ difficult near the crossing points-and recommends this system as an ideal testing ground for the study of chiral excitonic instability. A quantitative elaboration of the above results in Figs. 4 and 5 would be challenging and is delegated to future work, but may be achieved by considering frequency dependence of the self-energy beyond present phenomenological level (see Sec. IIB) or dealing with omitted contributions from its imaginary part. Incorporating higher-order fluctuations in interaction vertex may be also helpful, which are at the moment neglected in Eq. (9). Apart from excitonic pairing, one notes that so-far neglected spin-orbit interaction may also prefer gap opening at very low temperature and turn the system into a topological insulator $[81,82]$. Considering a subtle balance of these different mechanisms would be of particular interest, which may bridge the studies of correlated and topological materials at large.

\section{CONCLUSION}

In this paper, we have investigated excitonic instability of the continuum model for the pressurized organic conductor $\alpha$-(BEDT-TTF $)_{2} \mathrm{I}_{3}$, hosting two massless charge-neutral cones with a tilted dispersion relation. In particular, we have focused on the way a small charge off-neutrality and an inplane magnetic field affect the intervalley pairing instability, and analyzed how they impact on the transverse spin dynamics relevant to the precursor excitonic fluctuations. Considering the Coulomb interaction within realistic self-energy schemes using a renormalization-group approach, we have calculated the transverse spin susceptibility based on a ladder-type approximation coupled with a weak-coupling gap equation. We have found that electron-hole pairings are suppressed by a tiny doping due to degraded intervalley Fermi-surface nesting between the Zeeman-induced electron and hole pockets at the different cones, whereas they are stabilized by in-plane magnetic field because of enhanced nesting. Combined with additionally performed ${ }^{13} \mathrm{C}$-NMR experiments under pressure, we have shown that these nesting conditions directly affect precursor excitonic spin dynamics probed by the spinlattice relaxation rate, such that the spin fluctuations are sensitively suppressed as intervalley nesting is worsened upon doping. The presence of this tiny doping is in quantitative agreement with the earlier transport predictions in this system [53,63-65]. All these results lend good support to the notion that chiral excitonic instability and its precursor fluctuations provide a decent frame to understand excitations and dynamics near the crossing points in massless Dirac cones.

The characteristic instability of massless cones discussed here is directly linked to chiral property of the Hamiltonian, which is ubiquitous in various Dirac-Weyl semimetals for any dimension, pseudospin and symmetry $[3,4,76]$. Our framework to understand excitonic pairings and relevant precursor dynamics in titled Dirac cones may thus offer a generic platform for understanding excitonic instability in widespread topological materials

\section{ACKNOWLEDGMENTS}

The authors acknowledge G. Matsuno for helping with the modeling and coding; M. O. Goerbig, D. Basko, M. Baenitz, and H. Fukuyama for fruitful discussions; and M. Tamura and K. Miyagawa for providing samples and experimental support. Part of this work was performed at the High Field Laboratory for Superconducting Materials, Institute for Materials Research, Tohoku University. This work was supported by MEXT/JSPS KAKENHI under Grants No. 15K05166, No. 18H05225, No. 19J20677, and No. 19H01846.
[1] A. A. Abrikosov and S. D. Beneslavskii, Zh. Eksp. Teor. Fiz. 59, 1280 (1971) [Sov. Phys. JETP 32, 699 (1971)].

[2] J. González, F. Guinea, and M. A. H. Vozmediano, Nucl. Phys., Sect. B 424, 595 (1994).

[3] T. O. Wehling, A. M. Black-Schaffer, and A. V. Balatsky, Adv. Phys. 63, 1 (2014).

[4] V. N. Kotov, B. Uchoa, V. M. Pereira, F. Guinea, and A. H. Castro Neto, Rev. Mod. Phys. 84, 1067 (2012).

[5] W. Witczak-Krempa, G. Chen, Y. B. Kim, and L. Balents, Annu. Rev. Condens. Matter Phys. 5, 57 (2014).

[6] M. Dzero, J. Xia, V. Galitski, and P. Coleman, Annu. Rev. Condens. Matter Phys. 7, 249 (2016).

[7] H. Choi, W. Zhu, S. K. Cary, L. E. Winter, Z. Huang, R. D. McDonald, V. Mocko, B. L. Scott, P. H. Tobash, J. D. Thompson, S. A. Kozimor, E. D. Bauer, J. X. Zhu, and F. Ronning, Phys. Rev. B 97, 201114(R) (2018).

[8] J. González, F. Guinea, and M. A. H. Vozmediano, Phys. Rev. B 59, R2474(R) (1999).
[9] D. C. Elias, R. V. Gorbachev, A. S. Mayorov, S. V. Morozov, A. A. Zhukov, P. Blake, L. A. Ponomarenko, I. V. Grigorieva, K. S. Novoselov, F. Guinea, and A. K. Geim, Nat. Phys. 7, 701 (2011).

[10] M. Hirata, K. Ishikawa, K. Miyagawa, M. Tamura, C. Berthier, D. Basko, A. Kobayashi, G. Matsuno, and K. Kanoda, Nat. Commun. 7, 12666 (2016).

[11] M. Hirata, K. Ishikawa, G. Matsuno, A. Kobayashi, K. Miyagawa, M. Tamura, C. Berthier, and K. Kanoda, Science 358, 1403 (2017).

[12] J. E. Drut and T. A. Lähde, Phys. Rev. Lett. 102, 026802 (2009).

[13] J. González, Phys. Rev. B 82, 155404 (2010).

[14] O. V. Gamayun, E. V. Gorbar, and V. P. Gusynin, Phys. Rev. B 80, 165429 (2009).

[15] J. Sabio, F. Sols, and F. Guinea, Phys. Rev. B 81, 045428 (2010).

[16] O. O. Sobol, E. V. Gorbar, and V. P. Gusynin, Phys. Rev. B 88, 205116 (2013). 
[17] O. L. Berman, R. Y. Kezerashvili, and K. Ziegler, Phys. Rev. A 87, 042513 (2013).

[18] D. V. Khveshchenko, J. Phys.: Condens. Matter 21, 075303 (2009).

[19] E. V. Gorbar, V. P. Gusynin, V. A. Miransky, and I. A. Shovkovy, Phys. Rev. B 66, 045108 (2002).

[20] O. V. Gamayun, E. V. Gorbar, and V. P. Gusynin, Phys. Rev. B 81, 075429 (2010).

[21] D. V. Khveshchenko, Phys. Rev. Lett. 87, 246802 (2001).

[22] D. V. Khveshchenko and H. Leal, Nucl. Phys. B 687, 323 (2004).

[23] S. Raghu, X. L. Qi, C. Honerkamp, and S. C. Zhang, Phys. Rev. Lett. 100, 156401 (2008).

[24] I. F. Herbut, V. Juričić, and B. Roy, Phys. Rev. B 79, 085116 (2009).

[25] I. F. Herbut, Phys. Rev. Lett. 97, 146401 (2006).

[26] H. Wei, S. P. Chao, and V. Aji, Phys. Rev. Lett. 109, 196403 (2012).

[27] A. Sekine and K. Nomura, J. Phys. Soc. Jpn. 83, 094710 (2014).

[28] B. Roy, P. Goswami, and V. Juričić, Phys. Rev. B 95, 201102(R) (2017).

[29] A. Sekine and K. Nomura, Phys. Rev. B 90, 075137 (2014).

[30] J. Maciejko and R. Nandkishore, Phys. Rev. B 90, 035126 (2014).

[31] P. V. Buividovich, Phys. Rev. D 90, 125025 (2014).

[32] B. Roy and J. D. Sau, Phys. Rev. B 92, 125141 (2015).

[33] M. Laubach, C. Platt, R. Thomale, T. Neupert, and S. Rachel, Phys. Rev. B 94, 241102(R) (2016).

[34] C. Triola, A. Pertsova, R. S. Markiewicz, and A. V. Balatsky, Phys. Rev. B 95, 205410 (2017).

[35] A. Sharma, V. N. Kotov, and A. H. Castro Neto, Phys. Rev. B 95, 235124 (2017).

[36] P. Michetti and C. Timm, Phys. Rev. B 95, 125435 (2017).

[37] N. F. Mott, Philos. Mag. 6, 287 (1961).

[38] D. Jérome, T. M. Rice, and W. Kohn, Phys. Rev. 158, 462 (1967).

[39] Y. Wakisaka, T. Sudayama, K. Takubo, T. Mizokawa, M. Arita, H. Namatame, M. Taniguchi, N. Katayama, M. Nohara, and H. Takagi, Phys. Rev. Lett. 103, 026402 (2009).

[40] A. S. Rodin and A. H. Castro Neto, Phys. Rev. B 88, 195437 (2013).

[41] R. D. Pisarski, Phys. Rev. D 29, 2423 (1984).

[42] B. Wunsch, F. Guinea, and F. Sols, New J. Phys. 10, 103027 (2008).

[43] K. Asano and C. Hotta, Phys. Rev. B 83, 245125 (2011).

[44] I. L. Aleiner, D. E. Kharzeev, and A. M. Tsvelik, Phys. Rev. B 76, 195415 (2007).

[45] E. Uykur, W. Li, C. A. Kuntscher, and M. Dressel, Npj Quantum Mater. 4, 19 (2019).

[46] N. Tajima, S. Sugawara, M. Tamura, Y. Nishio, and K. Kajita, J. Phys. Soc. Jpn. 75, 051010 (2006).

[47] N. Tajima, S. Sugawara, R. Kato, Y. Nishio, and K. Kajita, Phys. Rev. Lett. 102, 176403 (2009).

[48] N. Tajima, M. Sato, S. Sugawara, R. Kato, Y. Nishio, and K. Kajita, Phys. Rev. B 82, 121420(R) (2010).

[49] D. Liu, K. Ishikawa, R. Takehara, K. Miyagawa, M. Tamura, and K. Kanoda, Phys. Rev. Lett. 116, 226401 (2016).

[50] K. Murata, K. Yokogawa, S. Arumugam, and H. Yoshino, Crystals 2, 1460 (2012).
[51] M. Sato, K. Miura, S. Endo, S. Sugawara, N. Tajima, K. Murata, Y. Nishio, and K. Kajita, J. Phys. Soc. Jpn. 80, 023706 (2011).

[52] T. Konoike, K. Uchida, and T. Osada, J. Phys. Soc. Jpn. 81, 043601 (2012).

[53] T. Konoike, M. Sato, K. Uchida, and T. Osada, J. Phys. Soc. Jpn. 82, 073601 (2013).

[54] H. Kino and T. Miyazaki, J. Phys. Soc. Jpn. 75, 034704 (2006).

[55] S. Katayama, A. Kobayashi, and Y. Suzumura, J. Phys. Soc. Jpn. 75, 054705 (2006).

[56] A. Kobayashi, S. Katayama, Y. Suzumura, and H. Fukuyama, J. Phys. Soc. Jpn. 76, 034711 (2007).

[57] S. Katayama, A. Kobayashi, and Y. Suzumura, Eur. Phys. J. B 67, 139 (2009).

[58] P. Alemany, J. P. Pouget, and E. Canadell, Phys. Rev. B 85, 195118 (2012).

[59] M. O. Goerbig, J. N. Fuchs, G. Montambaux, and F. Piéchon, Phys. Rev. B 78, 045415 (2008).

[60] F. Ghahari, H.- Y. Xie, T. Taniguchi, K. Watanabe, M. S. Foster, and P. Kim, Phys. Rev. Lett. 116, 136802 (2016).

[61] T. Tani and A. Kobayashi, J. Phys. Soc. Jpn. 88, 054713 (2019).

[62] H. Isobe and N. Nagaosa, J. Phys. Soc. Jpn. 81, 113704 (2012).

[63] N. Tajima, R. Kato, S. Sugawara, Y. Nishio, and K. Kajita, Phys. Rev. B 85, 033401 (2012).

[64] A. Kobayashi, Y. Suzumura, and H. Fukuyama, J. Phys. Soc. Jpn. 77, 064718 (2008).

[65] R. Kitamura, N. Tajima, K. Kajita, R. Kato, M. Tamura, T. Naito, and Y. Nishio, J. Phys. Soc. Conf. Proc. 1, 012097 (2014).

[66] M. Casula, P. Werner, L. Vaugier, F. Aryasetiawan, T. Miyake, A. J. Millis, and S. Biermann, Phys. Rev. Lett. 109, 126408 (2012).

[67] See Supplemental Material at http://link.aps.org/supplemental/ 10.1103/PhysRevResearch.2.033479 for supplementary text and figures.

[68] T. Sugano, G. Saito, and M. Kinoshita, Phys. Rev. B 34, 117 (1986).

[69] J. M. Luttinger and W. Kohn, Phys. Rev. 97, 869 (1955).

[70] A. Kobayashi, Y. Suzumura, H. Fukuyama, and M. O. Goerbig, J. Phys. Soc. Jpn. 78, 114711 (2009).

[71] R. Kondo, S. Kagoshima, N. Tajima, and R. Kato, J. Phys. Soc. Jpn. 78, 114714 (2009).

[72] A. Abragam, The Principles of Nuclear Magnetism (Clarendon, Oxford, 1961).

[73] G. Mahan, Many-Particle Physics (Springer, New York, 2000).

[74] A. L. Fetter and J. D. Walecka, Quantum Theory of ManyParticle Systems (McGraw-Hill, New York, 2003).

[75] A. Abrikosov, L. Gorkov, and I. Dzyaloshinskii, Methods of Quantum Field Theory in Statistical Physics (Dover, New York, 1975).

[76] M. O. Goerbig, Rev. Mod. Phys. 83, 1193 (2011).

[77] M. O. Goerbig (private communication).

[78] N. Tajima, Crystals 8, 126 (2018).

[79] A. Mori, M. Sato, T. Yajima, T. Konoike, K. Uchida, and T. Osada, Phys. Rev. B 99, 035106 (2019).

[80] J. Xue, J. Sanchez-Yamagishi, D. Bulmash, P. Jacquod, A. Deshpande, K. Watanabe, T. Taniguchi, P. Jarillo-Herrero, and B. J. Leroy, Nat. Mater. 10, 282 (2011).

[81] S. M. Winter, K. Riedl, and R. Valentí, Phys. Rev. B 95, 060404 (2017).

[82] T. Osada, J. Phys. Soc. Jpn. 87, 075002 (2018). 